\title{
Hypersensitivity Reaction to Paclitaxel: A Retrospective Analysis on the Non-Inferiority of Oral Famotidine to Intravenous Ranitidine in Premedication Regimen
}

Tsz Ting TSOI ( $\nabla$ tt696@ha.org.hk)

Tuen Mun Hospital

Wai Man YOUNG

Tuen Mun Hospital

Yin Ting CHEUNG

the Chinese University of Hong Kong

Tai Ning LAM

the Chinese University of Hong Kong

Chun Yu LAU

Princess Margaret Hospital

\section{Shing Yu SUEN}

Pamela Youde Nethersole Eastern Hospital

Yuk Tung Chanel TSUI

Queen Mary Hospital

Man Ho WONG

Prince of Wales Hospital

\section{Hong Kei WONG}

Queen Elizabeth Hospital

Tsz Hei TAM

United Christian Hospital

Keary Rui ZHOU

the Chinese University of Hong Kong

\section{Research Article}

Keywords: Hypersensitivity reactions, paclitaxel, premedication, histamine $\mathrm{H} 2$ receptor antagonists, famotidine, ranitidine

Posted Date: February 14th, 2022

DOI: https://doi.org/10.21203/rs.3.rs-1280524/v1

License: (c) (i) This work is licensed under a Creative Commons Attribution 4.0 International License. Read Full License 


\section{Abstract}

Background: Previous studies have only evaluated intravenous histamine $\mathrm{H} 2$ receptor antagonists for use as paclitaxel premedication. This study aims to investigate whether oral famotidine is non-inferior to intravenous ranitidine as a premedication to prevent paclitaxel-associated hypersensitivity reactions. It could provide guidance on the use of alternative orally administered agents.

Methods: It is a retrospective, multi-center, non-inferiority, historical control cohort study. Paclitaxel-naïve adult patients who began treatment with paclitaxel between August 2018 and December 2020, and received all cycles of paclitaxel in seven tertiary public hospitals in Hong Kong were enrolled. The patients received either intravenous ranitidine (control group) or oral famotidine (study group) as the premedication on the day of paclitaxel infusion. The primary outcome was the occurrence of a paclitaxel-induced hypersensitivity reaction of any severity during any cycle. The proportion of patients with the primary outcome in each group were compared by the two-proportion Z-test. The non-inferiority margin was pre-defined as an absolute hypersensitivity reaction rate difference of $5 \%$.

Results: We enrolled 1282 paclitaxel-naïve adult patients. Patients in both control $(n=635)$ and study $(n=647)$ groups had a median age of 59 years, and 476 (75\%) and 485 (75\%) were females, respectively. A median of five cycles of paclitaxel were administered in both groups. Hypersensitivity reactions occurred in 121 (19\%) patients in study group, and 105 (17\%) patients in control group (difference: $+2.2 \%$; 95\% confidence interval [Cl]: $-2.02 \%$ to $+6.35 \%$; non-inferiority one-sided $p=0.092$ ). A subgroup analysis of patients receiving standard-dose steroid premedication demonstrated a hypersensitivity reaction rate difference of $+0.4 \%(95 \% \mathrm{Cl}:-3.97 \%$ to $+4.83 \%$, noninferiority one-sided $p=0.021$ ).

Conclusions: We found no statistically significant differences in the hypersensitivity reaction rates between intravenous ranitidine and oral famotidine. Oral famotidine was not found to be non-inferior to intravenous ranitidine as a paclitaxel premedication based on the pre-defined non-inferiority margin. The use of oral famotidine with standard-dose steroid as premedication may provide better protection against hypersensitivity reactions.

\section{Background}

Paclitaxel is an antimitotic agent with broad activity and is used to treat ovarian cancer, breast cancer and nonsmall cell lung cancer ${ }^{1-3}$. However, its use was limited by a high incidence of hypersensitivity reactions (HSRs) (25 to $40 \%$ ) observed in some studies ${ }^{1,2,4,5}$.

A HSR is defined as any immunological response to a drug that results in an adverse reaction ${ }^{5}$. The severity of a HSR can be classified by the Common Terminology Criteria for Adverse Events (CTCAE) developed by the National Cancer Institute $(\mathrm{NCl})$ of the United States, with mild and transient reactions classified as Grade 1 and death classified as Grade $5^{6}$. The clinical manifestations of a HSR to paclitaxel are usually mild and transient, with flushing and rashes. However, severe reactions carry a mortality rate of $30 \%$, with respiratory and cardiovascular involvements ${ }^{1,5,7}$.

The use of premedication was investigated as a means to prevent HSRs to paclitaxel. A combination of corticosteroids, histamine $\mathrm{H} 1$ (H1RAs) and H2 receptor antagonists (H2RAs) has been shown to effectively suppress the HSR rate to $6-11 \%$, of which only $1-2 \%$ were severe cases ${ }^{3,7-9}$. Oral or intravenous (IV) dexamethasone $20 \mathrm{mg}$ given 12 and 6 hours before paclitaxel, in combination with IV diphenhydramine 50mg, and IV ranitidine $50 \mathrm{mg}$, IV cimetidine $300 \mathrm{mg}$, or IV famotidine $20 \mathrm{mg}$ given $30-60$ minutes before paclitaxel, is the 
currently recommended regimen ${ }^{1,3,7,10}$. Although single-dose IV dexamethasone $20 \mathrm{mg}$ has been suggested as a simplified corticosteroid regimen, it may result in higher HSR rates compared with the standard two-dose regimen ${ }^{5,11,12}$.

In 2019, ranitidine products were found contaminated with N-nitrosodimethylamine (NDMA), a probable human carcinogen, which led to interruption of global supply ${ }^{13-18}$. With the limited availability of H2RAs, the British Oncology Pharmacy Association issued a guidance document to review, rationalize, or avoid the use of H2RA for the prevention of HSR in anti-cancer therapy ${ }^{19}$. Other regulatory bodies also recommended alternative treatment options, including oral $\mathrm{H} 2 \mathrm{RAs} \mathrm{s}^{20,21}$. Small-scale studies found that ranitidine may have limited value as a paclitaxel HSR prophylactic agent ${ }^{22,23}$, but H2RA premedication remains part of the product license requirement for paclitaxel $^{18,19}$.

In Hong Kong, no other IV H2RAs were available following the recall of ranitidine in late September $2019^{24,25}$. The standard IV famotidine $20 \mathrm{mg}$ given 30 minutes before chemotherapy was selected as the comparator in a pharmacokinetics study ${ }^{26}$. It was found that oral famotidine $60 \mathrm{mg}$ given $1.5-2$ hours before chemotherapy resembled most closely to the pharmacokinetic profile of IV famotidine. The seven tertiary care acute public hospitals in Hong Kong subsequently adopted oral famotidine as the premedication regimen. The total replacement of IV ranitidine has been implemented for over one year. However, clinical evidence for alternative agents is limited, and oral H2RA agents have not been previously evaluated for use as premedication. Therefore, to fill the knowledge gap, we sought to investigate whether oral famotidine was non-inferior to IV ranitidine as a paclitaxel premedication to prevent HSRs.

\section{Methods}

\section{Setting}

This was a retrospective, multi-center, non-inferiority, historical control cohort study conducted in seven acute public hospitals in Hong Kong. Approval was granted from the Research Ethics Committees in the seven clusters of the Hospital Authority.

\section{Patients}

Eligible patients were $\geq 18$ years and paclitaxel-naïve, had received all cycles of paclitaxel at the study sites, and had received either IV ranitidine or oral famotidine as a premedication on the day of paclitaxel infusion. To fully investigate the effect of an agent, patients who experienced a change in H2RA premedication during any cycle of paclitaxel were excluded. Patients who received the nab-paclitaxel formulation were excluded from the study due to differences in pharmacokinetics and immunogenicity 7,27 .

\section{Procedures}

Patients who received paclitaxel were screened using the Clinical Data Analysis and Reporting System (CDARS). Electronic patient records (ePR) were reviewed to assess eligibility and collect basic demographic information, including sex, age, drug allergies, type of primary cancer, chemotherapy regimen, and premedication regimen. Additional information, including changes in drug regimens and descriptions and management of HSR episodes, was retrieved from the chemotherapy charts of enrolled patients. 
Enrolled patients who received IV ranitidine as a premedication were allocated to the control group, while those who received oral famotidine were allocated to the study group. Each hospital changed to the new premedication regimen on different dates between September and October 2019. To standardize the study period and maintain a 1:1 ratio between control and study patients, the patients in the control group received the first cycle of paclitaxel from August 2018 to September 2019, and patients in the study group began treatment from October 2019 to December 2020. Each case was followed until all cycles of paclitaxel had been administered.

\section{Premedication Regimens}

The full premedication regimens in all participating hospitals are shown in Additional file 1.

\section{H2RA}

The H2RA regimen in the control group was IV ranitidine $50 \mathrm{mg}$, while the study group received oral famotidine at either $40 \mathrm{mg}$ or $60 \mathrm{mg}$ on the day of paclitaxel infusion. In some hospitals, two oral doses of famotidine $20 \mathrm{mg}$ were administered to patients on the day before paclitaxel infusion.

\section{Corticosteroids}

Each dose of oral dexamethasone ranged from $8 \mathrm{mg}$ to $20 \mathrm{mg}$. At least two doses of dexamethasone were used for three-weekly paclitaxel regimens in most hospitals. Lower doses of corticosteroids were commonly used for weekly paclitaxel protocols or for later cycles if the patient does not experience any HSR in the earlier cycles.

As two doses of corticosteroids was recommended by most guidelines $3,7,10$, single dose steroid used as prophylaxis was categorized as low-dose steroid regimen. Prophylactic use of at least two doses of steroid was considered to be standard-dose regimens.

\section{H1RA}

The use of chlorpheniramine or diphenhydramine differed across the participating hospitals. Within each hospital, the same regimens were adopted in both three-weekly and weekly paclitaxel regimens.

\section{Outcomes}

The primary outcome was the occurrence of a paclitaxel-induced HSR of any severity in any cycle, as clinically diagnosed by the attending physician. The severity of HSR was classified by CTCAE version $5.0^{6}$. Subgroup analyses were performed according to sex, primary cancer, dose of paclitaxel, and premedication components.

Secondary outcomes included treatment discontinuation and switch to non-paclitaxel-based chemotherapy regimens and were analyzed by all-cause or paclitaxel-induced HSRs.

\section{Sample Size}

With an expected HSR rate of $10 \%$ in both the control and study groups ${ }^{7,9}$, a minimum of 566 patients per group were required to show non-inferiority of the primary outcome at a one-sided significance level of $2.5 \%$ and a power of $80 \%$.

\section{Statistical Analysis}


The proportion of patients with the primary outcome in each group were compared by the two-proportion Z-test. Considering the $5 \%$ variation in HSR rate (6-11\%) under standard premedication, and given that a $10 \%$ increase in the absolute HSR rate would approach the natural HSR rate (25-30\%), the non-inferiority margin of this study was pre-defined as an absolute HSR rate difference of $5 \%$. The exploratory secondary outcomes were tested by the twoproportion Z-test at a two-sided significance level of $5 \%$. Statistical analyses were performed using $\mathrm{R}$ statistical software (version 4.0.3) with the "ggplot2" and "gsDesign" packages.

\section{Results}

We enrolled 1,282 patients who received their first cycle of paclitaxel between August 9, 2018 and December 23, 2020. In the control group, 635 patients received IV ranitidine as a premedication in all cycles of paclitaxel; in the study group, 647 patients received oral famotidine in all cycles (Fig. 1).

\section{Baseline Characteristics}

\section{Patient Demographics}

Baseline characteristics were generally balanced between the two groups (Table 1). In both control and study group, $75 \%$ patients were females, with a median age of 59 years. A slightly higher proportion of patients in control group (17\%) reported a drug allergy when compared to the study group (12\%). The most common primary cancer types were breast cancer (control, 31\%; study, 28\%) and ovarian cancer (control, 20\%; study, 18\%), followed by lung cancer (control, 14\%; study, 18\%), endometrial cancer (control, 8\%; study, 11\%), and oesophageal cancer (control, 7\%; study, $6 \%)$. 
Table 1

Baseline Characteristics of Enrolled Patients

\begin{tabular}{|c|c|c|c|}
\hline & $\begin{array}{l}\text { Control Group } \\
\text { (IV Ranitidine) } \\
(n=635)\end{array}$ & $\begin{array}{l}\text { Study Group } \\
\text { (Oral Famotidine) } \\
(n=647)\end{array}$ & $P$ value \\
\hline Sex & & & 1.000 \\
\hline Male & $159(25 \%)$ & $162(25 \%)$ & \\
\hline Female & $476(75 \%)$ & $485(75 \%)$ & \\
\hline Age & $59(53-67)$ & $59(52-66)$ & 0.544 \\
\hline With known drug allergy & & & 0.022 \\
\hline Yes & $106(17 \%)$ & $79(12 \%)$ & \\
\hline No & $529(83 \%)$ & $568(88 \%)$ & \\
\hline \multicolumn{4}{|l|}{ Primary cancer types } \\
\hline Breast cancer & $196(31 \%)$ & $178(28 \%)$ & 0.189 \\
\hline Ovarian cancer & $123(20 \%)$ & $116(18 \%)$ & 0.508 \\
\hline Lung cancer & $91(14 \%)$ & $116(18 \%)$ & 0.080 \\
\hline Endometrial cancer & $50(8 \%)$ & $71(11 \%)$ & 0.058 \\
\hline Oesophageal cancer & $47(7 \%)$ & $38(6 \%)$ & 0.272 \\
\hline Cervical cancer & $28(4 \%)$ & $25(4 \%)$ & 0.624 \\
\hline Nasopharyngeal cancer & $29(5 \%)$ & $17(3 \%)$ & 0.062 \\
\hline Stomach cancer & $22(3 \%)$ & $23(4 \%)$ & 0.930 \\
\hline Others & $49(8 \%)$ & $63(10 \%)$ & 0.200 \\
\hline \multicolumn{4}{|l|}{ Chemotherapy regimens } \\
\hline Number of cycles received & $5(3-6)$ & $5(3-6)$ & 0.094 \\
\hline \multicolumn{4}{|l|}{ Frequency } \\
\hline Weekly & $121(19 \%)$ & $107(17 \%)$ & 0.239 \\
\hline 2-weekly & $22(4 \%)$ & $24(4 \%)$ & 0.814 \\
\hline 3-weekly & $489(77 \%)$ & $513(79 \%)$ & 0.323 \\
\hline 4-weekly & $3(0 \%)$ & $3(0 \%)$ & 1.000 \\
\hline Dose & & & 0.308 \\
\hline$<100 \mathrm{mg} / \mathrm{m}^{2}$ & $126(20 \%)$ & $114(18 \%)$ & \\
\hline
\end{tabular}

Data are $\mathrm{n}(\%)$ or median (IQR). Percentage may not add up to $100 \%$ due to rounding. 


\begin{tabular}{|c|c|c|c|}
\hline & $\begin{array}{l}\text { Control Group } \\
\text { (IV Ranitidine) } \\
(n=635)\end{array}$ & $\begin{array}{l}\text { Study Group } \\
\text { (Oral Famotidine) } \\
(n=647)\end{array}$ & $P$ value \\
\hline$\geq 100 \mathrm{mg} / \mathrm{m}^{2}$ & $509(80 \%)$ & $533(82 \%)$ & \\
\hline \multicolumn{4}{|c|}{ Premedication regimens } \\
\hline Steroid & & & 0.740 \\
\hline Standard-dose & $410(65 \%)$ & $412(64 \%)$ & \\
\hline Low-dose & $225(35 \%)$ & $235(36 \%)$ & \\
\hline H1RA & & & 0.810 \\
\hline Chlorpheniramine & $335(53 \%)$ & $337(52 \%)$ & \\
\hline Diphenhydramine & $300(47 \%)$ & $310(48 \%)$ & \\
\hline H2RA & & & / \\
\hline Ranitidine 50mg & $635(100 \%)$ & / & \\
\hline Famotidine 40mg & / & $145(22 \%)$ & \\
\hline Famotidine $60 \mathrm{mg}$ & / & $502(78 \%)$ & \\
\hline
\end{tabular}

\section{Chemotherapy Regimens}

As for chemotherapy regimens, $77 \%$ and $79 \%$ of patients received standard three-weekly paclitaxel in the control and study groups, respectively, followed by $19 \%$ and $17 \%$ patients on weekly paclitaxel. In both groups, the patients received a median of five cycles of paclitaxel.

Paclitaxel was commonly given at a standard dose of $175 \mathrm{mg} / \mathrm{m}^{2}$ every three weeks, or $80 \mathrm{mg} / \mathrm{m}^{2}$ weekly with other anti-cancer agents. The original prescribed doses in this study were either $45 \mathrm{mg} / \mathrm{m}^{2}-90 \mathrm{mg} / \mathrm{m}^{2}$ (lower spectrum), or $130 \mathrm{mg} / \mathrm{m}^{2}-260 \mathrm{mg} / \mathrm{m}^{2}$ (higher spectrum). After considering individual dose adjustments, we stratified patients by the paclitaxel dose level of $100 \mathrm{mg} / \mathrm{m}^{2}$. More patients received high paclitaxel dose $\left(\geq 100 \mathrm{mg} / \mathrm{m}^{2}\right)(\mathrm{control}, 80 \%$; study, $82 \%$ ) than the lower dose $\left(<100 \mathrm{mg} / \mathrm{m}^{2}\right)$ (control, $20 \%$; study, $18 \%$ ).

\section{Premedication Regimens}

The premedication regimens were similar between the control and study groups. Most patients (control, 65\%; study, $64 \%$ ) received standard-dose dexamethasone. The proportions of patients using chlorpheniramine (control, 53\%; study, 52\%) and diphenhydramine (control, 47\%; study, 48\%) as the H1RA were similar in both groups. For oral famotidine, $78 \%$ of the study patients received $60 \mathrm{mg}$, while the remaining $22 \%$ used a lower dose of $40 \mathrm{mg}$.

Occurrence of any paclitaxel-induced HSRs

Paclitaxel-induced HSRs occurred in 105 (17\%) of the 635 patients in the control group, and 121 (19\%) of the 647 patients in the study group (Table 2). An absolute risk difference of $+2.2 \%$ (95\% confidence interval [CI]: $-2.02 \%$ to 
$+6.35 \%$, non-inferiority one-sided $p=0.092$ ) was observed in the study group. Non-inferiority of oral famotidine to IV ranitidine could not be proved, as the upper bound of the $95 \% \mathrm{Cl}$ exceeded the pre-defined non-inferiority margin of $5 \%$.

\section{Subgroup analyses of HSR rates}

When stratified according to premedication regimen (Table 2), the HSR rate in patients who received low-dose corticosteroid (control, 26\%; study, 31\%) was more than two-fold of that in patients on standard-dose corticosteroid (control, $11 \%$; study, 12\%). Statistically significant non-inferiority of the study group was shown with standard-dose steroid $(+0.4 \%, 95 \% \mathrm{Cl}:-3.97 \%$ to $+4.83 \%$, non-inferiority one-sided $\mathrm{p}=0.021)$. For the H1RA agents, non-inferiority was demonstrated with diphenhydramine $(-1.3 \%, 95 \% \mathrm{Cl}:-7.60 \%$ to $+4.99 \%$, non-inferiority one-sided $\mathrm{p}=0.025)$ but not chlorpheniramine. Non-inferiority was only demonstrated with famotidine $40 \mathrm{mg}(-8.3 \%, 95 \% \mathrm{Cl}:-13.0 \%$ to $-2.08 \%$, non-inferiority one-sided $\mathrm{p}<0.001$ ) but not famotidine $60 \mathrm{mg}$.

Secondary outcomes

No significant differences were found between the two groups in all-cause treatment discontinuation (Table 2). The study group had a significantly lower rate of treatment discontinuation due to HSR from paclitaxel, which occurred in only $13(2 \%)$ patients, compared to 25 (4\%) patients in the control group $(-1.9 \% ; 95 \% \mathrm{Cl},-3.94 \%$ to $-0.0726 \%$; $\mathrm{p}=$ 0.021); however, the rate of therapy switch due to HSR from paclitaxel missed the threshold of statistical significance $(-1.5 \% ; 95 \% \mathrm{Cl},-3.34 \%$ to $+0.294 \%$; $=0.050)$. 
Table 2

Primary and Secondary Outcomes Analysis

\section{Primary Outcome: Occurrence of HSR}

\begin{tabular}{|c|c|c|c|c|}
\hline & $\begin{array}{l}\text { Control } \\
\text { Group } \\
\text { (IV } \\
\text { Ranitidine) } \\
(\mathrm{n}=635)\end{array}$ & $\begin{array}{l}\text { Study Group } \\
\text { (Oral } \\
\text { Famotidine) } \\
(n=647)\end{array}$ & $\begin{array}{l}\text { Absolute risk difference } \\
\text { (95\% Confidence Interval) }\end{array}$ & $\begin{array}{l}\text { Non- } \\
\text { inferiority } \\
\text { one-sided p }\end{array}$ \\
\hline Total & $105(17 \%)$ & $121(19 \%)$ & $+2.2 \%(-2.02 \%$ to $+6.35 \%)$ & 0.092 \\
\hline \multicolumn{5}{|l|}{ By sex } \\
\hline Male & $14(9 \%)$ & $21(13 \%)$ & $+4.2 \%(-2.76 \%$ to $+11.2 \%)$ & 0.404 \\
\hline Female & $91(19 \%)$ & $100(21 \%)$ & $+1.5 \%(-3.56 \%$ to $+6.55 \%)$ & 0.087 \\
\hline \multicolumn{5}{|c|}{ By primary cancer types ${ }^{a}$} \\
\hline Breast cancer & $36(18 \%)$ & $31(17 \%)$ & $-1.0 \%(-8.74 \%$ to $+6.95 \%)$ & 0.069 \\
\hline Ovarian cancer & $24(20 \%)$ & $30(26 \%)$ & $+6.3 \%(-4.28 \%$ to $+17.0 \%)$ & 0.599 \\
\hline Lung cancer & $14(15 \%)$ & $17(15 \%)$ & $-0.7 \%(-11.1 \%$ to $+9.01 \%)$ & 0.124 \\
\hline Endometrial cancer & $11(22 \%)$ & $18(25 \%)$ & $+3.4 \%(-12.6 \%$ to $+18.2 \%)$ & 0.416 \\
\hline Oesophageal cancer & $5(11 \%)$ & $4(11 \%)$ & $-0.1 \%(-13.9 \%$ to $+15.0 \%)$ & 0.231 \\
\hline \multicolumn{5}{|l|}{ By paclitaxel dose } \\
\hline$<100 \mathrm{mg} / \mathrm{m}^{2}$ & $17(13 \%)$ & $16(14 \%)$ & $+0.5 \%(-8.29 \%$ to $+9.61 \%)$ & 0.162 \\
\hline$\geq 100 \mathrm{mg} / \mathrm{m}^{2}$ & $88(17 \%)$ & $105(20 \%)$ & $+2.4 \%(-2.32 \%$ to $+7.13 \%)$ & 0.141 \\
\hline \multicolumn{5}{|l|}{ By premedication } \\
\hline \multicolumn{5}{|l|}{ Steroid } \\
\hline Standard-dose & $46(11 \%)$ & $48(12 \%)$ & $+0.4 \%(-3.97 \%$ to $+4.83 \%)$ & $0.021^{c}$ \\
\hline Low-dose & $59(26 \%)$ & $73(31 \%)$ & $+4.8 \%(-3.44-13.0 \%)$ & 0.485 \\
\hline \multicolumn{5}{|l|}{ H1RA } \\
\hline Chlorpheniramine & $45(13 \%)$ & $63(19 \%)$ & $\begin{array}{l}+5.3 \%(-0.295 \% \text { to } \\
+10.9 \%)\end{array}$ & 0.537 \\
\hline Diphenhydramine & $60(20 \%)$ & $58(19 \%)$ & $-1.3 \%(-7.60 \%$ to $+4.99 \%)$ & $0.025^{c}$ \\
\hline \multicolumn{5}{|l|}{ H2RA } \\
\hline Ranitidine 50mg & $105(17 \%)$ & & & \\
\hline Famotidine $40 \mathrm{mg}^{\mathrm{b}}$ & & $12(8 \%)$ & $-8.3 \%(-13.0 \%$ to $-2.08 \%)$ & $<0.001^{c}$ \\
\hline Famotidine $60 \mathrm{mg}^{\mathrm{b}}$ & & $109(22 \%)$ & $\begin{array}{l}+5.2 \%(+0.599 \% \text { to } \\
+9.86 \%)\end{array}$ & 0.530 \\
\hline
\end{tabular}




\begin{tabular}{|c|c|c|c|c|}
\hline \multicolumn{5}{|c|}{ Primary Outcome: Occurrence of HSR } \\
\hline \multicolumn{5}{|l|}{ Secondary Outcomes } \\
\hline & $\begin{array}{l}\text { Control } \\
\text { Group }\end{array}$ & $\begin{array}{l}\text { Study Group } \\
\text { (Oral } \\
\text { Famotidine) }\end{array}$ & Difference $(95 \% \mathrm{Cl})$ & Two-sided p \\
\hline & $\begin{array}{l}\text { (IV } \\
\text { Ranitidine) }\end{array}$ & $(n=647)$ & & \\
\hline & $(n=635)$ & & & \\
\hline \multicolumn{5}{|l|}{ Treatment discontinuation } \\
\hline All causes & $227(36 \%)$ & $242(37 \%)$ & $+1.7 \%(-3.62 \%$ to $+6.92 \%)$ & 0.731 \\
\hline Due to HSR to paclitaxel & $25(4 \%)$ & $13(2 \%)$ & $-1.9 \%(-3.94 \%$ to $-0.0726 \%)$ & $0.021^{c}$ \\
\hline \multicolumn{5}{|c|}{$\begin{array}{l}\text { Switch to non-paclitaxel based } \\
\text { chemotherapy regimens }\end{array}$} \\
\hline All causes & $109(17 \%)$ & $90(14 \%)$ & $-3.3 \%(-7.25 \%$ to $+0.713 \%)$ & 0.054 \\
\hline Due to HSR to paclitaxel & $21(3 \%)$ & $12(2 \%)$ & $-1.5 \%(-3.34 \%$ to $+0.294 \%)$ & 0.050 \\
\hline
\end{tabular}

a. Only top 5 cancer types were listed.

b. Compared to IV ranitidine

c. Statistically significant. Non-inferiority one-sided $p$ was significant below 0.025 , two-sided $p$ was significant below 0.05 .

Data are $\mathrm{n}(\%) ; \mathrm{HSR}=$ hypersensitivity reaction, IV = intravenous

Characteristics of HSR Episodes

Most characteristics of patients who experienced at least one HSR were similar to those at baseline (Table 3). Differences were observed for steroid premedication; $58 \%$ and $61 \%$ of the patients who had a HSR to paclitaxel were on low-dose dexamethasone in the control and study groups, compared to a baseline of only $35 \%$ and $36 \%$ of patients on low-dose regimens, respectively. 
Table 3

Characteristics of Patients with Hypersensitivity Reactions

\begin{tabular}{|c|c|c|}
\hline & $\begin{array}{l}\text { Control Group } \\
(n=105)\end{array}$ & $\begin{array}{l}\text { Study Group } \\
(n=121)\end{array}$ \\
\hline \multicolumn{3}{|l|}{ Sex } \\
\hline Male & $14(13 \%)$ & $21(17 \%)$ \\
\hline Female & $91(87 \%)$ & $100(83 \%)$ \\
\hline Age & $57(50-66)$ & $58(51-65)$ \\
\hline \multicolumn{3}{|l|}{ With known drug allergy } \\
\hline Yes & $16(15 \%)$ & $22(18 \%)$ \\
\hline No & $89(85 \%)$ & $99(82 \%)$ \\
\hline \multicolumn{3}{|l|}{ Primary cancer types } \\
\hline Breast cancer & $36(34 \%)$ & $31(26 \%)$ \\
\hline Ovarian cancer & $24(23 \%)$ & $29(24 \%)$ \\
\hline Lung cancer & $14(13 \%)$ & $17(14 \%)$ \\
\hline Endometrial cancer & $11(10 \%)$ & $17(14 \%)$ \\
\hline Oesophageal cancer & $5(5 \%)$ & $4(3 \%)$ \\
\hline Cervical cancer & $4(4 \%)$ & $4(3 \%)$ \\
\hline Nasopharyngeal cancer & $3(3 \%)$ & $3(2 \%)$ \\
\hline Stomach cancer & $3(3 \%)$ & $4(3 \%)$ \\
\hline Others & $5(5 \%)$ & $12(10 \%)$ \\
\hline \multicolumn{3}{|l|}{ Chemotherapy regimens } \\
\hline \multicolumn{3}{|l|}{ Frequency } \\
\hline Weekly & $18(17 \%)$ & $16(13 \%)$ \\
\hline 2-weekly & $5(5 \%)$ & $4(3 \%)$ \\
\hline 3-weekly & $82(78 \%)$ & $101(83 \%)$ \\
\hline \multicolumn{3}{|l|}{ Dose } \\
\hline$<100 \mathrm{mg} / \mathrm{m}^{2}$ & $19(18 \%)$ & $17(14 \%)$ \\
\hline$\geq 100 \mathrm{mg} / \mathrm{m}^{2}$ & $86(82 \%)$ & $104(86 \%)$ \\
\hline \multicolumn{3}{|l|}{ Premedication regimens } \\
\hline \multicolumn{3}{|l|}{ Steroid } \\
\hline Standard-dose & $44(42 \%)$ & $44(36 \%)$ \\
\hline
\end{tabular}




\begin{tabular}{|c|c|c|}
\hline & $\begin{array}{l}\text { Control Group } \\
(n=105)\end{array}$ & $\begin{array}{l}\text { Study Group } \\
(n=121)\end{array}$ \\
\hline Low-dose & $61(58 \%)$ & $74(61 \%)$ \\
\hline Nil & / & $1(1 \%)$ \\
\hline \multicolumn{3}{|l|}{ H1RA } \\
\hline Chlorpheniramine & $44(42 \%)$ & $63(52 \%)$ \\
\hline Diphenhydramine & $61(58 \%)$ & $58(48 \%)$ \\
\hline \multicolumn{3}{|l|}{$\mathrm{H} 2 \mathrm{RA}$} \\
\hline Ranitidine 50mg & 105 (100\%) & / \\
\hline Famotidine $40 \mathrm{mg}$ & I & $12(10 \%)$ \\
\hline Famotidine $60 \mathrm{mg}$ & l & 109 (90\%) \\
\hline \multicolumn{3}{|c|}{ Cycle number when first HSR occurred } \\
\hline 1 & $50(48 \%)$ & 57 (47\%) \\
\hline 2 & $40(38 \%)$ & $48(40 \%)$ \\
\hline 3 & $5(5 \%)$ & $11(9 \%)$ \\
\hline 4 & $6(6 \%)$ & $1(1 \%)$ \\
\hline 5 & $2(2 \%)$ & $1(1 \%)$ \\
\hline 6 & $2(2 \%)$ & $3(2 \%)$ \\
\hline
\end{tabular}


Table 3

Characteristics of Patients with Hypersensitivity Reactions (continued)

$\begin{array}{ll}\text { Control Group } & \text { Study Group } \\ (n=105) & (n=121)\end{array}$

\section{Severity}

Grade 1

Grade 2

48 (46\%)

$56(46 \%)$

Grade 3

47 (45\%)

$58(48 \%)$

Grade 4

$8(8 \%)$

$5(4 \%)$

Grade 5

$2(2 \%)$

$2(2 \%)$

0

0

\section{Recurrence}

Yes

32 (30\%)

42 (35\%)

No

49 (47\%)

65 (54\%)

No further cycles attempted

$24(23 \%)$

$14(12 \%)$

\section{Symptoms experienced}

Dermatologic

Flushing

55 (52\%)

78 (64\%)

Rash/ urticaria

36 (34\%)

$32(26 \%)$

Respiratory

Chest tightness

32 (30\%)

59 (49\%)

Dyspnea

$20(19 \%)$

$49(40 \%)$

Desaturation

$14(13 \%)$

$8(7 \%)$

Cardiovascular

Hypotension

$5(5 \%)$

$3(2 \%)$

Hypertension

$11(10 \%)$

$12(10 \%)$

Dizziness

$5(5 \%)$

$7(6 \%)$

Tachycardia

$11(10 \%)$

$8(7 \%)$

Bradycardia

$5(5 \%)$

$3(2 \%)$

Others

Abdominal pain

$3(3 \%)$

$3(2 \%)$

Data are $\mathrm{n}(\%)$ or median (IQR).

$\mathrm{HSR}=$ hypersensitivity reaction, $\mathrm{H} 1 \mathrm{RA}=$ histamine $\mathrm{H} 1$ receptor antagonist, $\mathrm{H} 2 \mathrm{RA}=$ histamine $\mathrm{H} 2$ receptor antagonist, IV = intravenous 


\begin{tabular}{|lll|}
\hline & $\begin{array}{l}\text { Control Group } \\
(\mathbf{n = 1 0 5 )}\end{array}$ & $\begin{array}{l}\text { Study Group } \\
(\mathbf{n}=\mathbf{1 2 1})\end{array}$ \\
\hline Back pain & $2(2 \%)$ & $3(2 \%)$ \\
\hline Nausea & $2(2 \%)$ & $1(1 \%)$ \\
\hline Treatment received & $36(34 \%)$ & $33(27 \%)$ \\
\hline Steroid & $40(38 \%)$ & $40(33 \%)$ \\
\hline Antihistamine (H1RA) & $2(2 \%)$ & $4(3 \%)$ \\
\hline Beta-2 agonist & $2(2 \%)$ & $2(2 \%)$ \\
\hline IV fluids & $18(17 \%)$ & $29(24 \%)$ \\
\hline Oxygen & $1(1 \%)$ & $1(1 \%)$ \\
\hline Inotropes & & \\
\hline Data are $n$ (\%) or median (IQR). & & \\
\hline $\begin{array}{l}\text { HSR = hypersensitivity reaction, H1RA = histamine H1 receptor antagonist, H2RA = histamine H2 receptor } \\
\text { antagonist, IV = intravenous }\end{array}$ & \\
\hline
\end{tabular}

Most HSR episodes occurred in the first two cycles of paclitaxel in both control (cycle 1, 48\%; cycle 2, 38\%) and study (cycle 1, 47\%; cycle 2, 40\%) groups. More than $90 \%$ of cases were Grade 1 (control: $46 \%$, study: $46 \%$ ) or Grade 2 (control: $45 \%$, study: $48 \%$ ) severity.

Recurrence of HSR occurred in $30 \%$ and $35 \%$ of the patients in the control and study groups respectively, although $23 \%$ and $12 \%$, respectively, did not attempt further cycles of paclitaxel following the occurrence of the first HSR.

The most commonly reported dermatologic symptoms were flushing (control, $52 \%$; study, $64 \%$ ) and rashes or urticaria (control, $34 \%$; study, $26 \%$ ); the respiratory system was also commonly involved, with patients reporting chest tightness (control, $30 \%$; study, 49\%), dyspnea (control, 19\%; study, $40 \%$ ), and even desaturation (control, $13 \%$; study, $7 \%$ ) in some cases. Cardiovascular involvements were less common.

Treatment with a steroid (control, $34 \%$; study, $27 \%$ ) or a $\mathrm{H} 1$ antihistamine (control, $38 \%$; study, $33 \%$ ) were usually required. Oxygen (control, $17 \%$; study, $24 \%$ ) was also given to address respiratory symptoms. More severe reactions required the use of IV fluids and inotropes.

Post-hoc Analysis

In view of the strong influence of variables other than the H2RA agent, a post-hoc analysis with logistic regression was performed to determine odds ratio of HSR occurrence, with corticosteroid premedication and the frequency of paclitaxel regimen as covariates (Table 4). Using oral famotidine did not have significant effects on HSR occurrence (odds ratio $1.14 ; 95 \% \mathrm{Cl}, 0.85$ to $1.53 ; \mathrm{p}=0.395)$. The use of low-dose corticosteroid premedication ( $\mathrm{p}<$ $0.001)$ and non-weekly paclitaxel regimens $(p<0.001)$ were associated with higher HSR rates. 
Table 4

Post-hoc Analysis: Factors Associated with Hypersensitivity Reaction Occurrence

\begin{tabular}{|c|c|c|c|}
\hline & $\begin{array}{l}\text { Odds Ratio of } \\
\text { HSR Occurrence }\end{array}$ & 95\% Confidence Interval & $P$ value \\
\hline \multicolumn{4}{|l|}{ Histamine $\mathrm{H} 2$ receptor antagonists } \\
\hline IV Ranitidine (reference) & / & / & \multirow[t]{2}{*}{0.395} \\
\hline Oral Famotidine & 1.14 & $0.85-1.53$ & \\
\hline \multicolumn{4}{|l|}{ Corticosteroid premedication } \\
\hline Low-dose corticosteroid (reference) & / & / & \multirow[t]{2}{*}{$<0.001$} \\
\hline Standard-dose corticosteroid & 0.26 & $0.19-0.35$ & \\
\hline \multicolumn{4}{|l|}{ Paclitaxel regimens } \\
\hline Weekly paclitaxel regimens (reference) & / & / & \multirow[t]{2}{*}{$<0.001$} \\
\hline Non-weekly paclitaxel regimens & 2.32 & $1.53-3.60$ & \\
\hline
\end{tabular}

\section{Discussion}

The overall HSR rate of $17-19 \%$ in our study was slightly higher than the reported incidence of about $10 \%$ in the literature, though the occurrence of severe Grade 3 to 5 reactions was low ${ }^{7,9}$. Although we were unable to demonstrate that oral famotidine was non-inferior to IV ranitidine as a premedication for preventing HSRs to paclitaxel, non-inferiority was shown in a subgroup analysis according to standard-dose steroid premedication.

Single-dose prophylaxis with dexamethasone to all paclitaxel regimens was adopted by two hospitals in this study. This simpler regimen was designed to increase compliance over the two-dose regimen with similar efficacy ${ }^{5,28}$; however, it was associated with higher HSR rates, possibly due to an inadequate level of immunosuppression ${ }^{11,12}$. Indeed, we also observed a markedly higher HSR rate in the low-dose steroid group, which may explain the high HSR rate in our study.

Influence of steroid premedication and paclitaxel regimen was controlled by logistic regression in the post-hoc analysis. With expected HSR rate at $10 \%$, a non-inferiority margin of $5 \%$ absolute risk difference would give an odds ratio of 1.588 as the upper threshold. In the analysis, upper bound of the study group was within this limit at 1.53. Using the standard steroid prophylactic regimen with oral famotidine may provide comparable protection to IV ranitidine. This post-hoc analysis was exploratory only and not powered. It would be worthwhile investigating the efficacy and safety of this regimen, and other oral H2RA agents with further studies. On the other hand, further use of the low-dose regimen should be re-considered given the high HSR incidence in this group.

Variations of other premedication components showed less impact. The other subgroups that showed noninferiority of oral famotidine included diphenhydramine and famotidine $40 \mathrm{mg}$. Although only diphenhydramine was recommended as the H1RA in the package insert ${ }^{3}$, differences in regional availability have meant that chlorpheniramine IV 10mg has been commonly used as an alternative H1RA with similar efficacy ${ }^{8,29-31}$. The 
apparent increase in HSR rate with chlorpheniramine could be attributed to its use with a low-dose corticosteroid. Similarly, given that all study hospitals adopted a regimen of standard-dose steroid with famotidine $40 \mathrm{mg}$, the result is likely to reflect the effect of the steroid rather than that of other premedication components. By pharmacokinetic simulations, an oral dose of $60 \mathrm{mg}$ should achieve the expected serum concentration better than $40 \mathrm{mg}^{26}$.

The secondary outcome analyses showed no significant differences in the rates of treatment discontinuation or therapy switch between the two groups. The switch from IV ranitidine to oral famotidine is unlikely to cause adverse clinical outcomes during the disease course.

In both groups, among patients who experienced HSRs due to paclitaxel, the severity was predominantly mild (Grades 1 or 2), and the recurrence rates were similar. Symptoms resolved soon after treatment with corticosteroid and $\mathrm{H} 1$ antihistamine. However, a larger proportion of patients in study group suffered from respiratory symptoms that required oxygen treatment as compared to the control group (control, 17\%; study, 24\%). Although this did not result in significantly higher rates of severe HSR in study group, more in-depth analyses in larger populations are needed.

As documented in the literature $9,10,27,32$, most HSRs occur in the first two cycles of paclitaxel infusion. Dose tapering or removal of steroid may occur after a few cycles of paclitaxel when no HSR is observed, especially in weekly paclitaxel regimens. This practice might have led to the development of HSRs in patients who had no previous reactions in earlier cycles. Following supply disruption of ranitidine, a committee of the National Health Service (NHS) in the United Kingdom even supported the practice of removing all premedication from the third cycle of weekly paclitaxel regimens ${ }^{33}$. Our findings suggest that clinicians should consider keeping steroid as part of the premedication regimen regardless of clinical HSR occurrence in all cycles of paclitaxel.

This study was not without limitations. First, the premedication regimens differ between hospitals due to differences in practice. Such variance might have resulted in a reduced power to detect non-inferiority in the final pooled analysis. Second, other clinical risk factors that may influence the outcome of HSR were not evaluated. For example, patients with atopy, a history of mild skin reactions in earlier treatments, respiratory dysfunction, and a postmenopausal status or obesity may be more prone to develop HSRs ${ }^{10,27}$. Third, the actual administration time of the premedication were not recorded. Most hospital protocols specified the exact administration time of oral famotidine before paclitaxel infusion but any deviations from the protocols were unknown. Other oral premedication may require self-administration by patients; therefore, HSRs due to non-adherence to premedication regimens may go unnoticed without supervision by clinicians. Finally, this retrospective analysis relied heavily on medical records, and under-reporting of mild reactions or incomplete record entries may have affected the quality of data.

\section{Conclusions}

In summary, the non-inferiority of oral famotidine to IV ranitidine as premedication to prevent HSRs to paclitaxel could not be proved. However, its non-inferiority in the subgroup with standard steroid prophylaxis was promising. Indeed, given the continued shortage of ranitidine, oral famotidine could be considered a viable alternative. Further studies should be conducted to assess the efficacy of oral H2RAs as a prophylactic agent for HSRs.

\section{Abbreviations}


HSR

Hypersensitivity reaction

CTCAE

Common Terminology Criteria for Adverse Events

$\mathrm{NCl}$

National Cancer Institute

H1RA

Histamine $\mathrm{H} 1$ receptor antagonist

H2RA

Histamine $\mathrm{H} 2$ receptor antagonist

IV

Intravenous

NDMA

$\mathrm{N}$-nitrosodimethylamine

CDARS

Clinical Data Analysis and Reporting System

ePR

Electronic patient records

NHS

National Health Service.

\section{Declarations}

\section{Ethics approval and consent to participate}

Ethics approval was granted from the Research Ethics Committees in the seven clusters of the Hospital Authority of Hong Kong, namely the Hong Kong East Cluster Ethics Committee, the Institutional Review Board of The University of Hong Kong / Hospital Authority Hong Kong West Cluster, the Research Ethics Committee (Kowloon Central / Kowloon East), the Kowloon West Cluster Research Ethics Committee, the Joint Chinese University of Hong Kong New Territories East Cluster Clinical Research Ethics Committee, and the New Territories West Cluster Clinical \& Research Ethics Committee. Informed consent was waived by all the above-named Ethics Committees for this retrospective study, which involved collection of existing data without active interventions performed to patients.

\section{Consent for publication}

Not applicable.

\section{Availability of data and materials}

All data generated or analysed during this study are included in this published article and its supplementary information files.

\section{Competing interests}

The authors declare that they have no competing interests.

\section{Funding}


No funding was received for this study.

\section{Authors' contributions}

Study design: All authors. Patient recruitment and analysis of individual study sites: TTT, SYS, CYL, YTT, THT, MHW, HKW. Statistical analysis, writing of manuscript: TTT. First review of manuscript: KRZ, TNL, YTC, CYL. Final approval of manuscript: All authors

\section{Acknowledgements}

We wished to thank Dr. C S Wong and Dr. T Y Ng from Department of Clinical Oncology, Tuen Mun Hospital, and Ms. Pauline Chu from Department of Pharmacy, Tuen Mun Hospital for their kind support of this study.

\section{References}

1. Rowinsky, E. K.; Donehower, R. C. Paclitaxel (Taxol). N. Engl. J. Med. 1995, 332 (15), 1004-1014. https://doi.org/10.1056/NEJM199504133321507.

2. Donehower, R. C. The Clinical Development of Paclitaxel: A Successful Collaboration of Academia, Industry and the National Cancer Institute. Stem Cells 1996, 14 (1), 25-28. https://doi.org/10.1002/stem.140025.

3. Taxol [Prescribing Information]. April 2011.

4. Boehm, D. K.; Maksymiuk, A. W. Paclitaxel Premedication Regimens. JNCl J. Natl. Cancer Inst. 1996, 88 (7), 463-465. https://doi.org/10.1093/jnci/88.7.463.

5. Zanotti, K. M.; Markman, M. Prevention and Management of Antineoplastic-Induced Hypersensitivity Reactions. Drug Saf. 2001, 24 (10), 767-779. https://doi.org/10.2165/00002018-200124100-00005.

6. National Cancer Institute. Common Terminology Criteria for Adberse Events (CTCAE), Version 5.0. November 27, 2017.

7. Picard, M.; Castells, M. C. Re-Visiting Hypersensitivity Reactions to Taxanes: A Comprehensive Review. Clin. Rev. Allergy Immunol. 2015, 49 (2), 177-191. https://doi.org/10.1007/s12016-014-8416-0.

8. Dewaele, E.; Verschueren, C.; Specenier, P. Premedication Strategy for Paclitaxel, Still an Unsolved Question after 30 Years. Belg. J. Med. Oncol. 2017, 11 (2), 46-55.

9. Otani, I. M.; Lax, T.; Long, A. A.; Slawski, B. R.; Camargo, C. A.; Banerji, A. Utility of Risk Stratification for Paclitaxel Hypersensitivity Reactions. J. Allergy Clin. Immunol. Pract. 2018, 6 (4), 1266-1273.e2. https://doi.org/10.1016/j.jaip.2017.08.025.

10. Sendo, T.; Sakai, N.; Itoh, Y.; Ikesue, H.; Kobayashi, H.; Hirakawa, T.; Nakano, H.; Oishi, R. Incidence and Risk Factors for Paclitaxel Hypersensitivity during Ovarian Cancer Chemotherapy. Cancer Chemother. Pharmacol. 2005, 56 (1), 91-96. https://doi.org/10.1007/s00280-004-0924-9.

11. Kloover, J. S.; den Bakker, M. A.; Gelderblom, H.; van Meerbeeck, J. P. Fatal Outcome of a Hypersensitivity Reaction to Paclitaxel: A Critical Review of Premedication Regimens. Br. J. Cancer 2004, 90 (2), 304-305. https://doi.org/10.1038/sj.bjc.6601303.

12. Kwon, J. S.; Elit, L.; Finn, M.; Hirte, H.; Mazurka, J.; Moens, F.; Trim, K. A Comparison of Two Prophylactic Regimens for Hypersensitivity Reactions to Paclitaxel. Gynecol. Oncol. 2002, 84 (3), 420-425. https://doi.org/10.1006/gyno.2001.6546. 
13. Food \& Drug Administration. Statement alerting patients and health care professional of NDMA found in samples of ranitidine https://www.fda.gov/news-events/press-announcements/statement-alerting-patientsand-health-care-professionals-ndma-found-samples-ranitidine.

14. European Medicines Agency. EMA to review ranitidine medicines following detection of NDMA https://www.ema.europa.eu/en/news/ema-review-ranitidine-medicines-following-detection-ndma.

15. Therapeutic Goods Administration. Ranitidine: TGA investigation - potential contamination with Nnitrosodimethylamine https://www.tga.gov.au/alert/ranitidine.

16. Health Sciences Authority. HSA Stops Supply of Eight Brands of Ranitidine Products in Singapore https://www.hsa.gov.sg/announcements/news/hsa-stops-supply-of-eight-brands-of-ranitidine-products-insingapore.

17. Health Canada. Health Canada assessing NDMA in ranitidine https://healthycanadians.gc.ca/recall-alertrappel-avis/hc-sc/2019/70989a-eng.php.

18. Walker, A.; Rudokas, M. Ranitidine Shortages Following International Recall- Implications on Pre-Medication Regimens to Prevent Hypersensitivity Reactions for Oncology Treatments.Pdf. 2020, 136920 Bytes. https://doi.org/10.6084/M9.FIGSHARE.13395944.V1.

19. British Oncology Pharmacy Association. Guidance on the Use of H2 Antagonists for the Prevention and Management of Hypersensitivity. June 2020.

20. Agence nationale de sécurité du médicament et des produits de santé. Recommandations pour l'utilisation des alternatives de la ranitidine https://ansm.sante.fr/actualites/recommandations-pour-lutilisation-desalternatives-de-la-ranitidine.

21. Food \& Drug Administration. FDA Requests Removal of All Ranitidine Products (Zantac) from the Market https://www.fda.gov/news-events/press-announcements/fda-requests-removal-all-ranitidine-products-zantacmarket.

22. Cox, J. M.; Van Doorn, L.; Malmberg, R.; Oomen-de Hoop, E.; Bosch, T. M.; van den Bemt, P. M. L. A.; Boere, I. A.; Jager, A.; Mathijssen, R. H.; van Leeuwen, R. W. F. 1851P The Added Value of the H2 Blocker Ranitidine in Premedication Regimens during Paclitaxel Treatment. Ann. Oncol. 2020, 31, S1060.

https://doi.org/10.1016/j.annonc.2020.08.1498.

23. Ryan, M.; Carrington, C. Sustaining Paclitaxel Administration in the Era of Ranitidineshortages [Oral Abstract]. Asia Pac. J. Clin. Oncol. 2020, 16 (S8), 59-60. https://doi.org/10.1111/ajco.13497.

24. The Government of the HKSAR. Recall of Zantac products (with photos) https://www.info.gov.hk/gia/general/201909/24/P2019092400771.htm.

25. Drug Office. Medicine recalls https://www.drugoffice.gov.hk/eps/do/en/consumer/safety_alerts_and_medical_recalls/medicine_recalls.html.

26. Cheng, A.; Yip, E.; Tsang, J.; Chan, P.; Choi, A.; Yiu, B.; Kam, J.; Young, G. W.; So, K. H.; Zuo, Z.; Cheung, Y. T.; Zhou, K.; Lam, T. T. N. PCN10 ORAL H2RA for Taxane Hypersensitivity Prevention: A Pharmacokinetic-Guided Decision. Value Health Reg. Issues 2020, 22, S6. https://doi.org/10.1016/j.vhri.2020.07.060.

27. Boulanger, J.; Boursiquot, J. N.; Cournoyer, G.; Lemieux, J.; Masse, M. S.; Almanric, K.; Guay, M. P.; Des pratiques en oncologie, T. C. de l'évolution. Management of Hypersensitivity to Platinum- and Taxane-Based Chemotherapy: Cepo Review and Clinical Recommendations. Curr. Oncol. 2014, 21 (4), 630. https://doi.org/10.3747/co.21.1966. 
28. Noronha, V.; Enting, D.; Thippeswamy, R.; Joshi, A.; Patil, V.; Prabhash, K. Hypersensitivity Reactions to Paclitaxel with a Modified Dexamethasone Intravenous Premedication Regimen. Cancer Res Stat Treat 2018, 1, 78-83.

29. Ueno, C.; Imada, M.; Sakai, Y.; Kashimoto, K.; Harada, Y.; Yamamoto, M. Effectiveness of D-Chlorpheniramine Maleate in Preventing Paclitaxel-Related Allergic Reactions. Iryo Yakugaku Jpn. J. Pharm. Health Care Sci. 2009, 35(11), 825-829. https://doi.org/10.5649/jjphcs.35.825.

30. Harada, T.; Doi, M.; Yamada, Y.; Akase, T. [Evaluation of short-time premedication with d-chlorpheniramine maleate injection for paclitaxel-induced hypersensitivity reaction]. Gan To Kagaku Ryoho 2008, 35 (8), 13471351.

31. NHS Northern Cancer Alliance. Policy for the Management of Allergic Reactions and/or Hypersensitivity Due to Chemotherapy and Monoclonal Antibodies. January 2018.

32. Banerji, A.; Lax, T.; Guyer, A.; Hurwitz, S.; Camargo, C. A.; Long, A. A. Management of Hypersensitivity Reactions to Carboplatin and Paclitaxel in an Outpatient Oncology Infusion Center: A 5-Year Review. J. Allergy Clin. Immunol. Pract. 2014, 2 (4), 428-433. https://doi.org/10.1016/j.jaip.2014.04.010.

33. NHS North Central London Joint Formulary Committee. Supply Disruption of Oral Ranitidine - Statement for NCL Trusts. June 15, 2020.

\section{Figures}




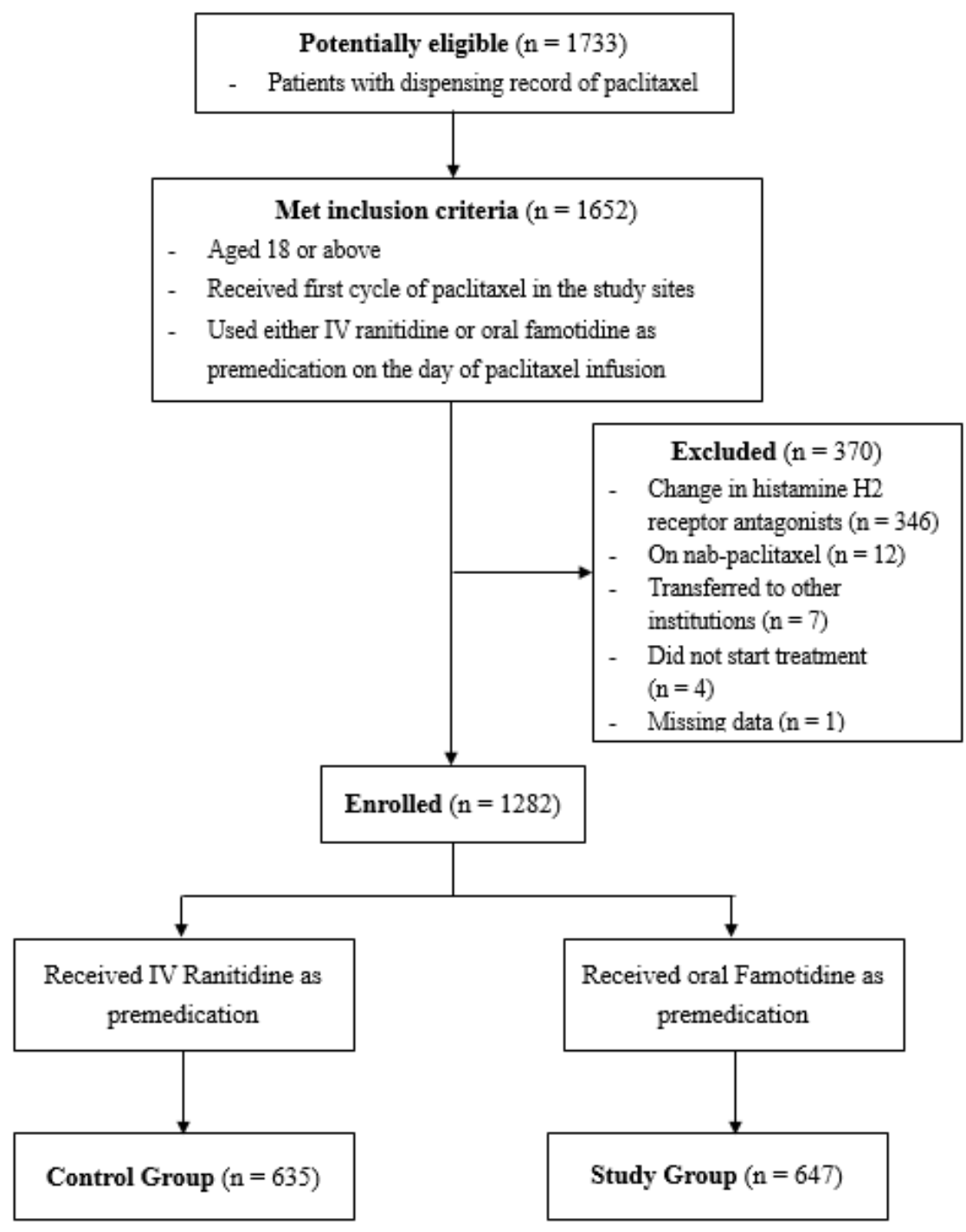

Figure 1

Study Flow Diagram

\section{Supplementary Files}

This is a list of supplementary files associated with this preprint. Click to download.

- Additionalfile1.docx

- Additionalfile2.xlsx 